\title{
Phosphorylation of Rictor at Thr1135 impairs the Rictor/Cullin-1 complex to ubiquitinate SGK1
}

\author{
Daming Gao*, Lixin Wan*, Wenyi Wei ${ }^{\bowtie}$ \\ Department of Pathology, Beth Israel Deaconess Medical Center, Harvard Medical School, Boston, MA 02215, USA \\ $\triangle$ Correspondence: wwei2@bidmc.harvard.edu
}

\begin{abstract}
The Rictor/mTOR complex plays a pivotal role in a variety of cellular functions including cellular metabolism, cell proliferation and survival by phosphorylating Akt at Ser473 to fully activate the Akt kinase. However, its upstream regulatory pathways as well as whether it has additional function(s) remain largely unknown. We recently reported that Rictor contains a novel ubiquitin E3 ligase activity by forming a novel complex with Cullin-1, but not with other Cullin family members. Furthermore, we identified SGK1 as its downstream target. Interestingly, Rictor, but not Raptor or mTOR, promotes SGK1 ubiquitination. As a result, SGK1 expression is elevated in Rictor $^{-/}$MEFs. We further defined that as a feedback mechanism, Rictor can be phosphorylated by multiple AGC family kinases including Akt, S6K and SGK1. Phosphorylation of Rictor at the Thr1135 site did not affect its kinase activity towards phosphorylating its conventional substrates including Akt and SGK1. On the other hand, it disrupted the interaction between Rictor and Cullin-1. Consequently, T1135E Rictor was defective in promoting SGK1 ubiquitination and destruction. This finding further expands our knowledge of Rictor's function. Furthermore, our work also illustrates that Rictor E3 ligase activity could be governed by specific signaling kinase cascades, and that misregulation of this process might contribute to SGK overexpression which is frequently observed in various types of cancers.
\end{abstract}

KEYWORDS mTORC2, Rictor, kinase, E3 ubiqutin ligase, Cullin-1, SGK1

\section{RICTOR-CONTAINING MTORC2 COMPLEX IS A SER/THR PROTEIN KINASE}

Mammalian target of rapamycin (mTOR), belongs to the serine/threonine kinase of the phosphatidylinositol kinaserelated family, and is conserved in a broad range of eukaryotic organisms (Sabatini, 2006; Wullschleger et al., 2006). It was recently established that the $\mathrm{mTOR}$ kinase plays a critical role in a wide spectra of cellular homeostasis, cellular growth and survival pathways. This is achieved mainly by acting as a sensor mechanism to receive upstream inputs from multiple growth-promoting signals and then transducing them to the downstream effector signaling pathways (Reiling and Sabatini, 2006; Guertin and Sabatini, 2007). By associating with different subset of components, the mTOR kinase could assemble into at least two different complexes that are termed as mTOR complex 1 (mTORC1) and mTOR complex 2 (mTORC2) (Reiling and Sabatini, 2006; Guertin and Sabatini, 2007). The mTORC1 complex contains the subunits mTOR, Raptor, PRAS40 and $\mathrm{mLST8/G} \beta \mathrm{L}$, and was reported to phosphorylate a faculty of downstream targets including S6K and the 4E-BP protein. Phosphorylation by mTORC1 leads to activation of S6K and inhibition of 4E-BP, thereby augmenting the protein translation process (Yang and Guan, 2007). mTORC1 function has also been implicated in regulating cell growth and autophagy. On the other hand, the mTORC2 complex is composed of mTOR, Rictor, $\mathrm{mLST} 8 / \mathrm{G} \beta \mathrm{L}$, PRTOR/PRR5 and Sin1 (Fig. 1A) (Jacinto et al., 2006; Shiota et al., 2006). Unlike mTORC1, the mTORC2 complex is relatively insensitve to short-term rapamycin treatment, although more recent work demonstrated that prolonged rapamycin treatment also dissociated the mTORC2 complex

"These authors contributed equally to this work. 
(Sarbassov et al., 2006). The assembled mTORC2 complex phosphorylates the hydrophobic motif of Akt (Ser473) (Sarbassov et al., 2005) and SGK1 (Ser422) (GarciaMartinez and Alessi, 2008), leading to the full activation of these two kinases. Since aberrant activation of Akt is a hallmark of many types of cancers (Manning and Cantley, 2007), hyperactivation of mTORC2 activity has been implicated in cancer progression (Guertin and Sabatini, 2007).

Previous work clearly demonstrated that mTORC1 activity is tightly controlled in vivo. In response to growth factors, activation of the PI3K kinase pathway leads to subsequent phosphorylation of the TSC2 and PRAS40 proteins by Akt, thus leading to activation of the mTORC1 kinase (Manning and Cantley, 2007). Recent work demonstrated that the activity of mTORC1 can also be stimulated by the Rag GTPase in response to nutrient stimulation (Sancak et al., 2008). In addition to the positive regulation, mTORC1 is also subject to layers of negative regulation. For example, phosphorylation of Raptor by AMP-activated protein kinase (AMPK) in response to low energy state represses mTORC1 activity (Gwinn et al., 2008). Recent studies also revealed that Raptor could form a complex with Cullin-4 and that the proteasome pathway might be critical for its kinase activity (Ghosh et al., 2008). This work indicated that Raptor might be multi-functional, which contains activities other than its kinase activity. Compared to the accumulated knowledge of mTORC1 regulation, very little is known so far regarding the regulatory mechanism for activation of $\mathrm{mTORC2}$, although it is a critical upstream pathway governing Akt1 activity. Unlike Raptor, which is shown to be a WD40-repeat containing protein, Rictor contains no characterized functional domain. However, Rictor was reported to associate with the Cullin 4 complex (Ghosh et al., 2008) as well. Nevertheless, the additional function(s) of Rictor remains largely unknown.

\section{RICTOR FORMS A NOVEL E3 LIGASE COMPLEX WITH CULLIN-1 AND RBX1, AND PHOSPHORYLATION OF RICTOR AT T1135 IMPAIRS ITS E3 LIGASE ACTIVITY}

We obtained experimental evidence for a novel function of the Rictor protein, which is promoting the ubiquitination and destruction of SGK1 (Gao et al., 2010). Thus far the major research focus of Rictor has been in identifying its downstream phosphorylation targets, and how this affects their functions. Although Rictor has been indicated to be associated with Cullin-4 (Ghosh et al., 2008), its role as an E3 ligase has not been formally presented. We found that by specific association with Cullin 1 and Rbx1, Rictor can form a functional E3 ubiquitin ligase complex (Fig. 1B) (Gao et al., 2010). We further defined that this activity of Rictor does not involve mTOR and other mTORC2 components such as Sin-1 and G $\beta$ L. This indicates that the kinase activity of Rictor and the E3 ligase activity of Rictor could possibly be separated. Consistent with this notion, we showed that phosphorylation of Rictor at T1135 minimally affects the kinase activity of Rictor, but severely diminished its E3 ligase activity (Fig. 2). In an effort to further define the minimal motif present in Rictor critical for its kinase or E3 ligase activity, we found that both $\mathrm{N}$ terminus and C-terminus of Rictor is required for proper interaction with mTOR (Huang et al., 2009; Gao et al., 2010). This indicates that the formation of the multi-component mTORC2 complex requires an intact Rictor protein. On the other hand, although deletion of neither the $\mathrm{N}$-terminus nor the C-terminus of Rictor affects its interaction with Cullin 1 , the resulting Rictor/Cullin 1 complex is defective in promoting SGK1 ubiquitination (Gao et al., 2010). It is highly possible that it requires the presence of both the $\mathrm{N}$ terminus and C-terminus to maintain the Cullin/Rictor complex in an active conformation. However, more studies are needed to fully understand the structural and functional difference and relationship between the kinase and E3 ligase activity of Rictor, and to elucidate the other possible components of the Rictor/Cullin-1 complex (Fig. 1A and 1B).

It seems likely that by associating with mTOR, mLST8/ G $\beta$ L, PRTOR/PRR5 and Sin1, Rictor can form a kinase complex. On the other hand, Rictor can interact with Cullin-1, $\mathrm{Rbx} 1$ and other unknown factors to form an E3 ligase complex. Although we found that mTOR is not involved in its E3 ligase activity, it still remains unclear whether these two complexes share some common scaffolding proteins and what the crosstalk is between these two complexes. Recent studies revealed that many other kinases possess E3 ligase activity as well. For example, MEKK1 acts as an E3 ligase to promote ERK1 destruction (Lu et al., 2002), while the DYRK2 protein can form an E3 ligase to promote katanin p60 ubiquitination (Maddika and Chen, 2009). Since many ubiquitination processes require a prior phosphorylation event for efficient substrate recognition, it has been shown that by coupling both the kinase and the E3 ligase activity in adjacency provides for more efficient destruction. For example, the Skp2 E3 ligase is in tight association with the Cyclin A/Cdk2 complex and this is thought to promote the destruction of its substrate, $\mathrm{p} 27$, which is required to be phosphorylated by Cyclin A/Cdk2 (Carrano et al., 1999). However, we found that the phosphorylation of SGK1 by Rictor at S422 did not serve to promote its subsequent ubiquitination by Rictor (data not shown). It is possible that there are unknown Rictor ubiquitination substrate(s) that require prior phosphorylation by Rictor. It is thus critical to define Rictor ubiquitin substrates other than SGK1, which will help further reveal the in vivo physiological role of Rictor for cell growth and development. 


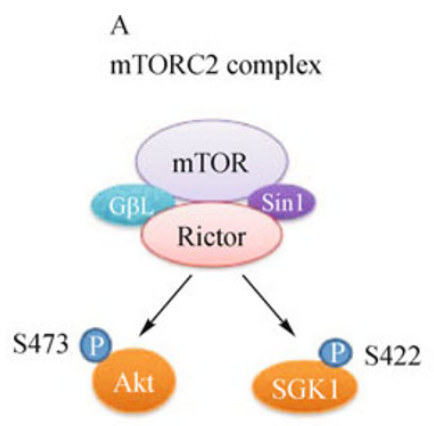

B

Rictor-cullin1-Rbxl complex

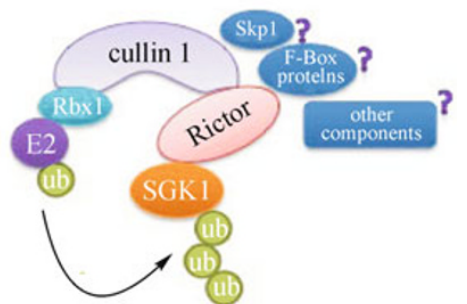

Figure 1. By association with a distinct subset of co-factors, Rictor can acquire either kinase or E3 ubiquitin ligase activity. (A) In association with mTOR, Sin1 and G $\beta$ L, Rictor could form the mTORC2 complex, which is a Ser/Thr protein kinase complex that could phosphorylate Akt at Ser473 and SGK at Ser422, leading to their full activation. (B) Rictor could form a novel complex with Cullin-1 and Rbx1 to promote SGK1 ubiquitination. It remains unclear whether Skp1, F-box or other unidentified components are also involved in SGK1 ubiquitination.

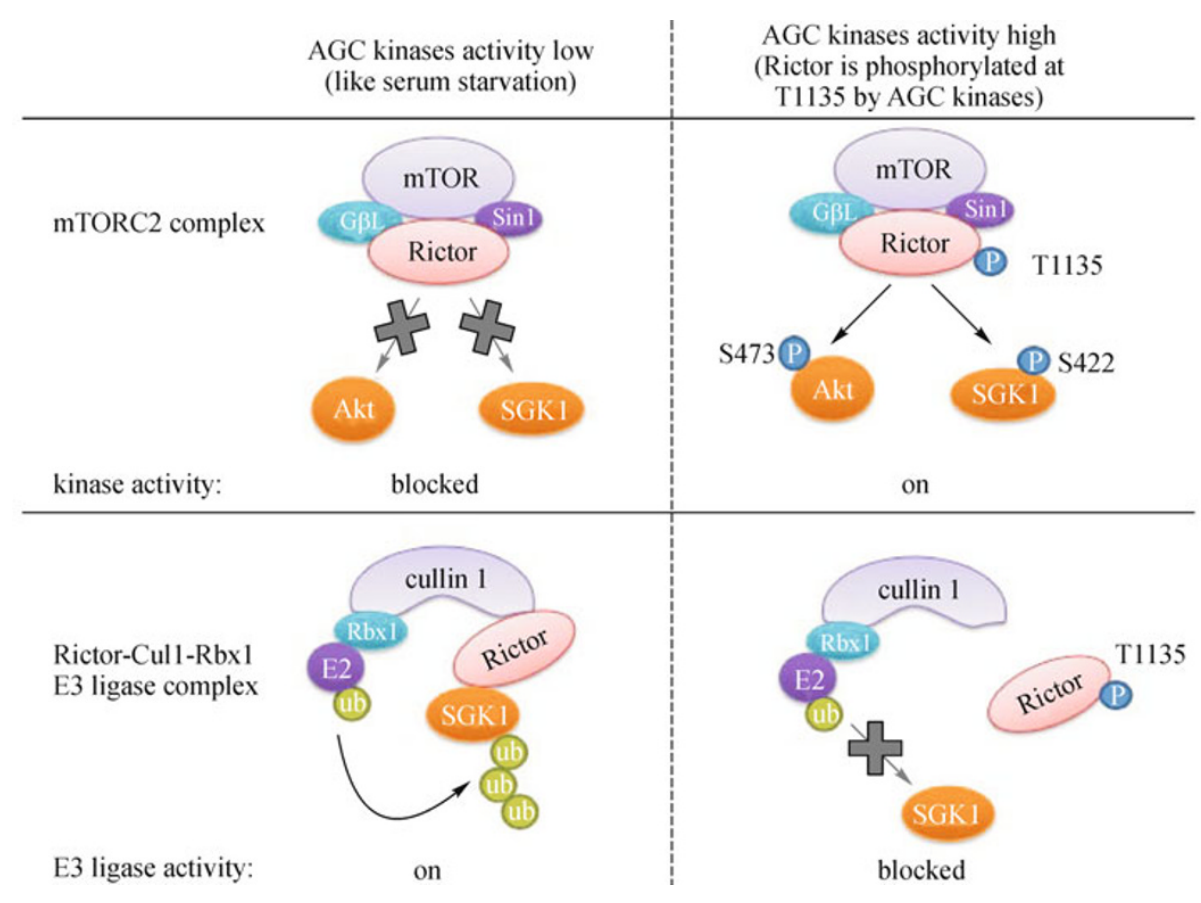

Figure 2. Phosphorylation of Rictor at Thr1135 minimally affects the mTORC2 kinase activity, but severely impairs the E3 ligase activity of the Rictor/Cullin-1 complex. In low AGC kinase activity conditions (such as serum starvation condition), mTORC2 activity is low. As a result, neither Akt nor SGK is in its activated state, and the majority of Rictor is not phosphorylated at Thr1135. Non-phosphorylated Rictor forms a complex with Cullin-1 and Rbx-1 to promote SGK1 ubiquitination and degradation. In high AGC kinase activity conditions, mTORC2 complex activity is high, leading to phosphorylation of Akt at Ser473 and SGK1 at Ser422. This modification fully activates both Akt and SGK1, both of which are capable of phosphorylating Rictor at Thr1135. Phosphorylation of Rictor at Thr1135 dissociates Rictor from Cullin-1, thus leading to inactivation of the Rictor/Cullin-1 E3 ligase complex and stabilization of the SGK1 protein.

\section{CROSSTALK BETWEEN SGK1 AND RICTOR SETS UP A POSITIVE FEEDBACK LOOP ALLOWING SGK1 ACCUMULATION AFTER MITOGENIC STIMULATION}

Furthermore, we identified that a variety of AGC family of kinases including SGK1, Akt and S6K could potentially affect the novel E3 ligase activity of Rictor by disrupting the Rictor/ Cullin-1 complex through phosphorylation of Rictor at the T1135 site. On one hand, this provides another piece of evidence about the complex regulatory mechanisms that control the mTOR pathway. On the other hand, it further indicates that besides activating Akt and SGK1 through complexing with mTOR, Rictor also regulates cell signaling pathways by controlling the stability of key signaling 
components like SGK1 (Gao et al., 2010). SGK1 activity has been demonstrated previously to be activated by the PI3K kinase pathway (Park et al., 1999; Loffing et al., 2006). Although SGK1 has been shown to be degraded by other E3 ligases including Nedd4-2 and CHIP, it remains largely unknown how the destruction of SGK1 by Nedd4-2 or CHIP is regulated and whether this process is related to PI3K kinase activity. Our results offer a novel point of view for the regulation of SGK1 in the setting of the PI3K kinase signaling pathway. SGK1 is very unstable in normal conditions, and upon serum or growth factor induction, acute activation of the PI3K kinase pathway, which subsequently activates the Akt and S6K kinases, might also contribute to the induction of SGK1 stability by disrupting the Rictor/Cullin-1 complex. Furthermore, since SGK1 could also negatively regulate Rictor E3 ligase activity without affecting its kinase activity, this results in a positive feedback loop to boost the SGK1 activity shortly post-stimulation. However, more studies should be performed to investigate whether the ability of Nedd4-2 and CHIP to degrade SGK1 is also affected by the $\mathrm{PI} 3 \mathrm{~K} /$ Akt pathway.

\section{MISREGULATION OF RICTOR/CULLIN-1- MEDIATED SGK1 DESTRUCTION MIGHT CONTRIBUTE TO FREQUENT SGK1 OVEREXPRESSION IN CANCERS}

Our results further implicate that aberrant activation of the $\mathrm{PI3K} /$ Akt pathway, which is a hallmark of many types of cancers, might contribute to impair Rictor/Cullin-1-mediated ubiquitination of SGK1, thus contributing to the frequent elevation of SGK1 in many cancers. There are many reported ways to activate the PI3K/Akt pathway, which include loss-offunction mutations in the PTEN tumor suppressor, as well as gain-of-function mutations in upstream regulators such as the receptors HER2, EGF-R and Ras (Majumder and Sellers, 2005). In addition, there is also a high frequency of constitutively active PI3K mutations identified in several human cancers (Samuels et al., 2004). We found that depletion of endogenous PTEN resulted in elevated phosphorylation of Rictor at Thr1135, presumably through activation of either the Akt or S6K kinase. Subsequently, we found elevated SGK1 expression (Gao et al., 2010). SGK1 overexpression has been reported in multiple cancers including breast cancer. Interestingly, although SGK1 has been suggested to have redundant functions with Akt, simultaneous overexpression of both Akt1 and SGK1 has been reported in many breast cancers (Sahoo et al., 2005). Our work suggested that since SGK1, but not Akt, is subject to the ubiquitination by the Rictor/Cullin-1 complex, elevated Akt activity might block the function of Rictor/Cullin-1, leading to accumulation of SGK1. Thus, our work implicates a novel regulatory pathway for SGK1, which might provide a mechanistic explanation for the frequent overexpression of SGK1 in multiple cancers.

Collectively, our results provide novel insight into how Rictor governs SGK1 abundance and activity by promoting its ubiquitination and subsequent destruction. We further define a feedback mechanism that could terminate the E3 ligase activity of the Rictor/Cullin-1 complex through phosphorylation of Rictor at the T1135 site to dissociate Cullin-1 and Rictor interaction. On one hand, our finding provides novel functional insight for Rictor, a key regulator of cell metabolism and cell growth. On the other hand, we also offer a novel mechanistic insight into SGK1 stability controlled by the PTEN/PI3K/Akt signaling pathway, which are hotspot mutation targets in cancers, and how misregulation of this process will possibly contribute to SGK1 overexpression in cancers.

\section{ACKNOWLEDGEMENTS}

We thank Alan Lau, Hiroyuki Inuzuka and Pengda Liu for critical reading of the manuscript, and other members of the Wei laboratory for useful discussions. Wenyi Wei is a Kimmel Scholar, V Scholar and Karin Grunebaum Cancer Research Foundation Fellow. This work was supported in part by the DOD Prostate New Investigator award to W.W., and NIH grant GM089763 to W.W.

\section{REFERENCES}

Carrano, A.C., Eytan, E., Hershko, A., and Pagano, M. (1999). SKP2 is required for ubiquitin-mediated degradation of the CDK inhibitor p27. Nat Cell Biol 1, 193-199.

Gao, D., Wan, L., Inuzuka, H., Berg, A.H., Tseng, A., Zhai, B., Shaik, S., Bennett, E., Tron, A.E., Gasser, J.A., et al. (2010). Rictor forms a complex with Cullin-1 to promote SGK1 ubiquitination and destruction. Mol Cell 39, 797-808.

Garcia-Martinez, J.M., and Alessi, D.R. (2008). mTOR complex 2 (mTORC2) controls hydrophobic motif phosphorylation and activation of serum- and glucocorticoid-induced protein kinase 1 (SGK1). Biochem J 416, 375-385.

Ghosh, P., Wu, M., Zhang, H., and Sun, H. (2008). mTORC1 signaling requires proteasomal function and the involvement of CUL4-DDB1 ubiquitin E3 ligase. Cell Cycle 7, 373-381.

Guertin, D.A., and Sabatini, D.M. (2007). Defining the role of mTOR in cancer. Cancer Cell 12, 9-22.

Gwinn, D.M., Shackelford, D.B., Egan, D.F., Mihaylova, M.M., Mery, A., Vasquez, D.S., Turk, B.E., and Shaw, R.J. (2008). AMPK phosphorylation of raptor mediates a metabolic checkpoint. Mol Cell 30, 214-226.

Huang, J., Wu, S., Wu, C.L., and Manning, B.D. (2009). Signaling events downstream of mammalian target of rapamycin complex 2 are attenuated in cells and tumors deficient for the tuberous sclerosis complex tumor suppressors. Cancer Res 69, 61076114.

Jacinto, E., Facchinetti, V., Liu, D., Soto, N., Wei, S., Jung, S.Y., Huang, Q., Qin, J., and Su, B. (2006). SIN1/MIP1 maintains rictormTOR complex integrity and regulates Akt phosphorylation and substrate specificity. Cell 127, 125-137.

Loffing, J., Flores, S.Y., and Staub, O. (2006). Sgk kinases and their 
role in epithelial transport. Annu Rev Physiol 68, 461-490.

Lu, Z., Xu, S., Joazeiro, C., Cobb, M.H., and Hunter, T. (2002). The PHD domain of MEKK1 acts as an E3 ubiquitin ligase and mediates ubiquitination and degradation of ERK1/2. Mol Cell 9 , 945-956.

Maddika, S., and Chen, J. (2009). Protein kinase DYRK2 is a scaffold that facilitates assembly of an E3 ligase. Nat Cell Biol 11, 409-419.

Majumder, P.K., and Sellers, W.R. (2005). Akt-regulated pathways in prostate cancer. Oncogene 24, 7465-7474.

Manning, B.D., and Cantley, L.C. (2007). AKT/PKB signaling: navigating downstream. Cell 129, 1261-1274.

Park, J., Leong, M.L., Buse, P., Maiyar, A.C., Firestone, G.L., and Hemmings, B.A. (1999). Serum and glucocorticoid-inducible kinase (SGK) is a target of the PI 3-kinase-stimulated signaling pathway. EMBO J 18, 3024-3033.

Reiling, J.H., and Sabatini, D.M. (2006). Stress and mTORture signaling. Oncogene 25, 6373-6383.

Sabatini, D.M. (2006). mTOR and cancer: insights into a complex relationship. Nat Rev Cancer 6, 729-734.

Sahoo, S., Brickley, D.R., Kocherginsky, M., and Conzen, S.D. (2005). Coordinate expression of the PI3-kinase downstream effectors serum and glucocorticoid-induced kinase (SGK-1) and Akt-1 in human breast cancer. Eur J Cancer 41, 2754-2759.
Samuels, Y., Wang, Z., Bardelli, A., Silliman, N., Ptak, J., Szabo, S., Yan, H., Gazdar, A., Powell, S.M., Riggins, G.J., et al. (2004). High frequency of mutations of the PIK3CA gene in human cancers. Science 304, 554.

Sancak, Y., Peterson, T.R., Shaul, Y.D., Lindquist, R.A., Thoreen, C. C., Bar-Peled, L., and Sabatini, D.M. (2008). The Rag GTPases bind raptor and mediate amino acid signaling to mTORC1. Science 320, 1496-1501.

Sarbassov, D.D., Ali, S.M., Sengupta, S., Sheen, J.H., Hsu, P.P., Bagley, A.F., Markhard, A.L., and Sabatini, D.M. (2006). Prolonged rapamycin treatment inhibits mTORC2 assembly and Akt/PKB. Mol Cell 22, 159-168.

Sarbassov, D.D., Guertin, D.A., Ali, S.M., and Sabatini, D.M. (2005). Phosphorylation and regulation of Akt/PKB by the rictor-mTOR complex. Science 307, 1098-1101.

Shiota, C., Woo, J.T., Lindner, J., Shelton, K.D., and Magnuson, M.A. (2006). Multiallelic disruption of the rictor gene in mice reveals that mTOR complex 2 is essential for fetal growth and viability. Dev Cell 11, 583-589.

Wullschleger, S., Loewith, R., and Hall, M.N. (2006). TOR signaling in growth and metabolism. Cell 124, 471-484.

Yang, Q., and Guan, K.L. (2007). Expanding mTOR signaling. Cell Res $17,666-681$. 